\section{Notch nicked again}

\section{By Lev Osherovich, Senior Writer}

When preclinical studies last year suggested that broadly inhibiting signaling through the Notch pathway runs the risk of triggering tumors in endothelial tissue, two companies-Roche's Genentech Inc. unit and Aveo Pharmaceuticals Inc. - tried to sidestep the adverse effects of pan-Notch inhibition by selectively targeting Notch 1. Now, a new paper from Washington University in St. Louis researchers suggests that Notch 1 inhibition may also cause tumors. ${ }^{1}$

Although some academics say the entire class of therapeutics should be abandoned, at least one company-OncoMed Pharmaceuticals Inc. - thinks it's still business as usual because the gene knockout data that underpin the new study do not agree with the company's preclinical results. Indeed, whether the mouse model used by the Washington University team has any bearing on the preclinical Notch 1 (NOTCH1) mAbs at Genentech and Aveo is a point of debate.

The Notch pathway emerged in the 1990s as an attractive target for eliminating the stem cell-like tumor progenitor cells thought to drive many endothelial and lymphatic cancers. Starting with a 1997 licensing deal on Notch
"It's enough to remove just one receptorNOTCH1 - and you have toxicity. This says that the therapeutic window for drugs that target NOTCH1 is extremely small."

-Raphael Kopan, Washington University in St. Louis
This says that the therapeutic window for drugs that target NOTCH1 is extremely small."

\section{Notch knockout}

Previous efforts to study the effect of disrupting NOTCH1 were stymied by the protein's essential role in embryonic development. To get around this problem, Kopan's team engineered a mouse in which the Notch1 gene was present throughout embryogenesis but became conditionally knocked out in adults.

Compared with Notch1 heterozygotes, the conditional Notch1 knockouts had excessive vascularization throughout the body and had vascular endothelial tumors in the liver. The Notch1-deficient mice also had shorter life spans than heterozygote controls.

Data were reported in The Journal of Clinical Investigation.

"Endothelial cells must have NOTCH1 signaling to prevent them from proliferating uncontrollably," said Kopan. Thus, he thinks that the findings do not bode well for therapeutics that target NOTCH1.

Because Notch pathway inhibitors aim to block angiogenesis, the increased angiogenesis seen in Kopan's mice seems counterintuitive. However, previous findings suggest that blood vessels are highly sensitive to Notch pathway signals and can proliferate abnormally if there is either too little or too much activity in the pathway.

Spyros Artavanis-Tsakonas, professor of cell biology at Harvard Medical School, said that extrapolating from Kopan's genetic model of Notch 1 to NOTCH1-targeting mAbs in the clinic is tricky. For instance, it's unclear targets between Exelixis Inc. and Yale University, companies have been trying to inhibit various components of the pathway in hopes of blocking both tumor growth and vascularization. ${ }^{2}$

One of the most promising targets was delta-like ligand 4 (DLL4), an extracellular ligand that activates all four mammalian Notch receptors. However, a Genentech team last year reported that antibodies against DLL4 induced liver and vascular endothelial cell proliferation in mice, rats and monkeys. ${ }^{3}$

At that time, researchers at Genentech and Exelixis hypothesized that the DLL4 mAb's side effects could result from its effect on all four receptors.

Genentech subsequently reoriented its program toward mAbs that selectively block NOTCH1 or NOTCH2 and later in 2010 reported that such antibodies block tumor vascularization and growth without eliciting intestinal endothelial tumors. ${ }^{4}$

The new report from the Washington University team led by Raphael Kopan, professor of molecular biology and medicine, suggests that inactivating Notch 1 in mice may cause abnormal proliferation of endothelial tissue, leading to vascular tumors, particularly in the liver.

"We have shown mechanism-based toxicity," said Kopan. "It's enough to remove just one receptor-Notch1—and you have toxicity. whether NOTCH1 mAbs would cause a sufficiently severe reduction in Notch signaling to elicit the same effects as the knockout.

"Notch signaling is one of those pathways that are exceedingly dosage dependent," said Artavanis-Tsakonas. "If you lose Notch signaling, you change the fate of cells. There's absolutely no doubt that if you start fiddling with Notch signaling there will be consequences."

Artavanis-Tsakonas, who cloned the fly homolog of NOTCH1 in the 1980s, is a cofounder of Exelixis. That biotech licensed patents on Artavanis-Tsakonas' Notch pathway discoveries and subsequently completed a three-year collaboration with Genentech to develop agents against targets in the pathway.

"When you make a genetic knockout, what you get is not necessarily reflective of what you get with partial inhibition with a drug for a brief period of time," said Max Wicha, professor of oncology at the University of Michigan Medical School and director of the University of Michigan Comprehensive Cancer Center.

Wicha is a cofounder of OncoMed and a principal investigator on the Phase Ib solid tumor trial of the company's OMP-21M18, a DLL4-targeting $\mathrm{mAb}$ that is partnered with GlaxoSmithKline plc.

Regeneron Pharmaceuticals Inc. and sanofi-aventis Group also have a mAb targeting DLL4, REGN421, in Phase I testing for solid tumors. 
Wicha said the side effects seen in Kopan's complete and permanent genetic knockout might not be recapitulated by a mAb that partially decreases Notch signaling.

Timothy Hoey, SVP of cancer biology at OncoMed, made a similar argument. "Sometimes antibodies and knockouts aren't the same in terms of phenotypes," he said.

Hoey added that different animal models of Notch1 signaling have yielded divergent results, so it's unclear to what extent Kopan's mouse findings mirror what may occur in humans.

He also noted that in last year's report on the effect of Notch1directed mAbs, "the Genentech team reported vascular neoplasms in rats, but this was not observed in monkeys."

\section{Shorter is better}

One thing that everyone agrees on is that long-term treatment with Notch pathway inhibitors is likely to cause trouble.

Kopan said long-term blockade of Notch signaling already has yielded safety problems in the clinic. As an example, he pointed to Eli Lilly and Co.'s semagacestat (LY450139), a $\gamma$-secretase inhibitor that blocks Notch signaling via a different mechanism than Notch- or DLL4-targeting mAbs. The compound failed to meet its endpoint in a Phase III trial in Alzheimer's disease (AD) and encountered multiple safety problems, including increased rates of skin cancer compared with placebo. ${ }^{5}$

"In a chronic setting, inhibiting NOTCH1 would be a problem," said Kopan. "Anybody on these drugs for a year should be watched closely."

Wicha agreed. "The strategy for Notch inhibitors is to combine them with chemotherapy intermittently and not continuously," he said. "I'd be very worried about long-term chronic therapy."

Wicha is also principal investigator of a Phase I trial of MK-0752, a $\gamma$-secretase inhibitor from Merck \& Co. Inc., in combination with docetaxel to treat advanced breast cancer.

Artavanis-Tsakonas noted that some of the proliferative side effects of Notch pathway inhibition could be caused by inflammation, so glucocorticoids or other anti-inflammatory agents could be helpful as adjunct therapies.

OncoMed's Hoey said the company's current trials will not be derailed by Kopan's findings. In addition to the DLL4 inhibitor OMP21M18, OMP-59R5, a mAb that targets NOTCH2 and NOTCH3, is in Phase I testing for solid tumors.
"We're developing mAbs for patients with advanced pancreatic cancer where patients have a year or less to live. We're not contemplating treating somebody for 20 years," he said.

Genentech and Aveo declined to comment on Kopan's study.

Kopan's team is now exploring an alternative approach to inhibiting Notch pathway signaling in lymphatic tumors by blocking the dimerization of the Notch intracellular domain, which drives the transcriptional effects of Notch signaling. That approach, he said, may have a more subtle effect on Notch pathway activity than outright inhibition.

Aileron Therapeutics Inc. has a peptide antagonist of the Notch intracellular domain in preclinical testing to treat $\mathrm{T}$ cell acute lymphoblastic leukemia (T-ALL).

Osherovich, L. SciBX 4(6); doi:10.1038/scibx.2011.151

Published online Feb. 10, 2011

\section{REFERENCES}

1. Liu, Z. et al. J. Clin. Invest.; published online Feb. 1, 2011; doi:10.1172/JCl43114

Contact: Raphael Kopan, Washington University in St. Louis

School of Medicine, St. Louis, Mo. e-mail: Kopan@wustl.edu

2. Fulmer, T. SciBX 3(17); doi:10.1038/scibx.2010.512

3. Yan, M. et al. Nature 463, E6-E7 (2010)

4. Wu, Y. et al. Nature 464, 1052-1057 (2010)

5. Osherovich, L. BioCentury 18(37), A9-A11; Aug. 23, 2010

\section{COMPANIES AND INSTITUTIONS MENTIONED}

Aileron Therapeutics Inc., Cambridge, Mass.

Aveo Pharmaceuticals Inc. (NASDAQ:AVEO), Cambridge, Mass.

Eli Lilly and Co. (NYSE:LLY), Indianapolis, Ind.

Exelixis Inc. (NASDAQ:EXEL), South San Francisco, Calif.

Genentech Inc., South San Francisco, Calif.

GlaxoSmithKline plc (LSE:GSK; NYSE:GSK), London, U.K.

Harvard Medical School, Boston, Mass.

Merck \& Co. Inc. (NYSE:MRK), Whitehouse Station, N.J.

OncoMed Pharmaceuticals Inc., Redwood City, Calif.

Regeneron Pharmaceuticals Inc. (NASDAQ:REGN), Tarrytown, N.Y.

Roche (SIX:ROG; OTCQX:RHHBY), Basel, Switzerland

sanofi-aventis Group (Euronext:SAN; NYSE:SNY), Paris, France

University of Michigan Comprehensive Cancer Center,

Ann Arbor, Mich.

University of Michigan Medical School, Ann Arbor, Mich.

Washington University in St. Louis, St. Louis, Mo.

Yale University, New Haven, Conn. 